\title{
A Study on the Effect of CSV on the Performance of Partner Companies: An Analysis of Artificial Neural Networks
}

\author{
Won-seok Bang1), Sun-Hwa Kim²), Kuk-Hoan Wee³)
}

\begin{abstract}
This study investigates the effectiveness of creating shared value (CSV), which is attracting attention as a new value creation and an element that enables continuous management of a company or an organization. The purpose of this study is to identify the effects of economic and social values, which are sub-factors of shared value creation (CSV), on the performance of partner companies. To this end, a total of 246 cases collected after surveys were conducted for 50 small and medium-sized companies' employees and practical personnel in the aviation complex. Based on the validity and reliability of all questionnaires applied to the variables in this study, the artificial neural networks (ANN) was used to test the hypotheses. The ANN, a new analysis method, shows the nonlinear relationship between variables rather than linear regression analysis model or a structural equation model that represents simple linear results. The results showed that economic value, one of the sub-factors of the creation of shared values, has a positive effect on the performance of partner companies. In addition, social value, the other sub-factor of the creation of shared values, was found to have a positive effect on the performance of partner companies. The results of this study demonstrated a non-linear relationship between two related variables. Therefore, the academic practical implications are very meaningful.

Keywords: Creating Shared Value (CSV), Economic Value, Social Value, Partner Company's Performance, Artificial Neural Networks (ANNs)
\end{abstract}

\section{Introduction}

Considering the environment of fierce competition and uncertainty environment surrounding the company, "sustainable management" requires a change in the strategy and perspective of a company that creates shared values with partners and has insights into social problems, needs, and deficiencies.

Recently, using CSV, it has become possible to create a new ecosystem that can create transformative innovation, i.e. destroy existing ecosystems and create shared values. Therefore, companies need a CSV design that creates transformative innovations that ensure long-term environmental sustainability[1].

However, not only global companies, but also some domestic companies are using CSV as a corporate strategy; however, they experience difficulties due to the lack of theoretical foundation for CSV and ambiguity about the direction and method for appropriate CSV

Received(March 30, 2020), Review Result(1st: April 29, 2020, 2nd: June 17, 2020), Accepted(June 25, 2020)

1) (Professor, First Author) 52725 Dept. Business, Gyeongnam Science and Technology Univ., Chiram-dong, Jinju-si, Gyeongsangnam-do, Korea

email: bangws@daum.net

2) (Student, Co Author), 48434 Dept. Education, Kyungsung University, Daeyeon 3(sam)-dong, Nam-gu, Busan, Korea

email: yama6161@naver.com

3) (Student, Corresponding Author) 48513 Dept. Industry, Pukyong National Univ., Daeyeon 3(sam)-dong, Nam-gu, Busan Korea

email: wkh64@pusan.ac.kr 
activities.

Therefore, this study examines the previous studies of CSV to evaluate the effects of economic values and social values, which are sub-factors of CSV, on the performance of partner companies. To this end, we intend to present meaningful theoretical and practical implications through the analysis of artificial neural networks (ANN), a new research method.

\section{Literature Review}

\subsection{CSV (Creating Shared Value)}

For companies that play an important role in society today, the center of management's role in society is being studied from a variety of viewpoints, spanning together including economic, legal, social, and environmental perspectives (Jose, 2016). From a new point of view, CSV activity attracts attention as a strategy for competitive advantage of a company.

Due to the limitations of the existing corporate social responsibility (CSR) activities, the company needs a new management strategy; in this context, creating shared value (CSV), which can simultaneously create the economic and social values of the company, has attracted a considerable attention[2]. At the heart of the CSV strategy is value creation. Shared value refers to the sharing of economic and social values[3].

Even in the case of large companies, finding and sharing new values through collaboration with SMEs, which are partner companies, is an important factor in sustainable management. In this context, CSV focused on the role of a company to create new values in social and business relationships. At the heart of the CSV strategy is value creation. Shared value refers to the sharing of economic and social values[3] (see [Fig. 1]).

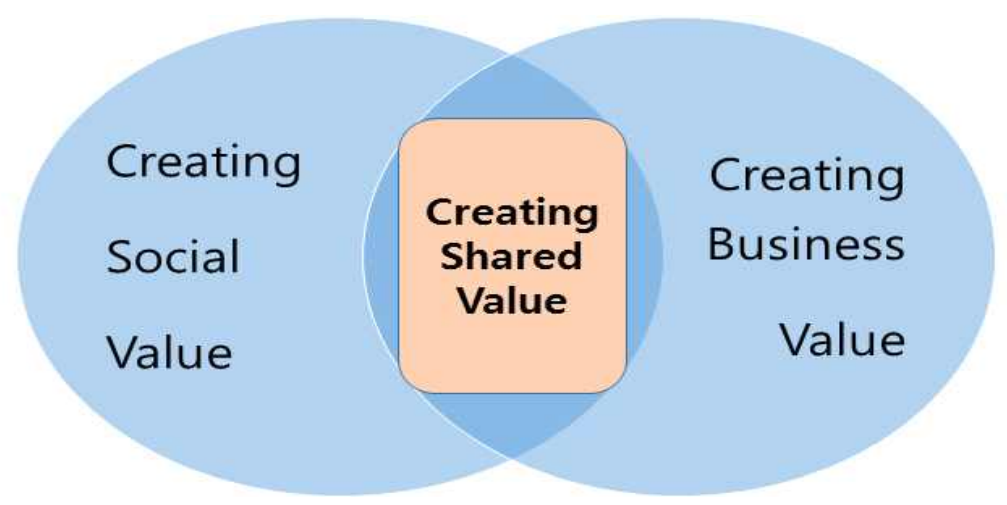

[Fig. 1] Concept of CSV

Porter and Kramer (2011) proposed the following three mechanisms for CSV: (1) products that meet customer needs, (2) value chain, and (3) regional cluster development[4]. Said differently, it creates new shared values in the value chain with products or suppliers and partner companies that can meet the potential needs of customers, or creates values that can create social performance through community problem solving and cluster development. This kind of a company can gain a competitive advantage. Therefore, company decision-makers have acknowledged that environmental, social, and governance activities create value for the 
company, and this shared value activity provides new opportunities for sustainable economic growth of the company.

The concept of CSV based on the theory of stakeholders that it must meet the expectations of all stakeholders in order to promote sustainability is in line with the Bible saying, "Love your neighbor as yourself do" (Matthew 19: 19).

From a business manager's perspective, if you engage in management with all your stakeholders like your neighbor, you will be interested in CSV that value not only economic values, but also social values[3].

\subsection{Relationship between CSV and Partner Companies' Performance}

A company's CSV activities can increase its effectiveness by creating positive shared values among stakeholders, particularly among partner companies and society.

In $B$ to $B$ relations between companies, performance is classified into the following three categories: attitude performance, behavioral performance, and economic performance.

Economic value and social value activities, which are sub-factors of CSV for large companies, will improve the performance of partner companies. Based on these considerations, the following two hypotheses were formulated:

Hypothesis1 Economic value activities would have a positive effect on the performance of business partners.

Hypothesis2 Social value activities would have a positive effect on partner companies' performance.

\subsection{Artificial Neural Networks}

Artificial Neural networks are nowadays used in diverse research field including business, engineering, etc.[5]. For instance, an ANN is a methodological approach for predicting COVID-19 epidemic[6], and one of the effective analysis methodologies to accurately forecast the demand[7].

Based on the bioelectrical activity of the brain's biological model, especially the neurons in the brain, an artificial neural network (ANN) is a sub-field of machine learning within the field of artificial intelligence research, and the goal of ANN research is to develop a machine learning system[8]. Neural networks perform multiple recognition through simple connections between different components. The same layer, one or more processing unit(s) are called artificial neurons that produce a simplified version of the brain's neurons, the role of neurons in the human brain to process and analyze data using mathematical processes in an ANN[9].

Each neural network has three main neural layers. The first layer of the input layer connects the network with the people processing the data and passes it to the processor. The second layer is a hidden layer, whose function takes data from the input layer and takes into account the weight of the relationship between other units and the hidden layer.

These weights determine when the hidden layer should be activated. The last layer is the output layer. The function of this layer depends on the weight between the hidden and output 
units and the activation of the hidden layer. The model performs forecasting analysis based on multi-layer feed-forward neural network with back propagation.

\section{Research Model and Method}

\subsection{Research Model}

The model of this study starts with the question of whether the CSV factor, which is composed of the sub-factors of economic and social values, affects the performance of SMEs' partner companies. Said differently, the research model was set up as shown in [Fig. 2] to understand the effects of CSV sub-factors, economic and social value, on the performance of SMEs for aviation manufacturers.

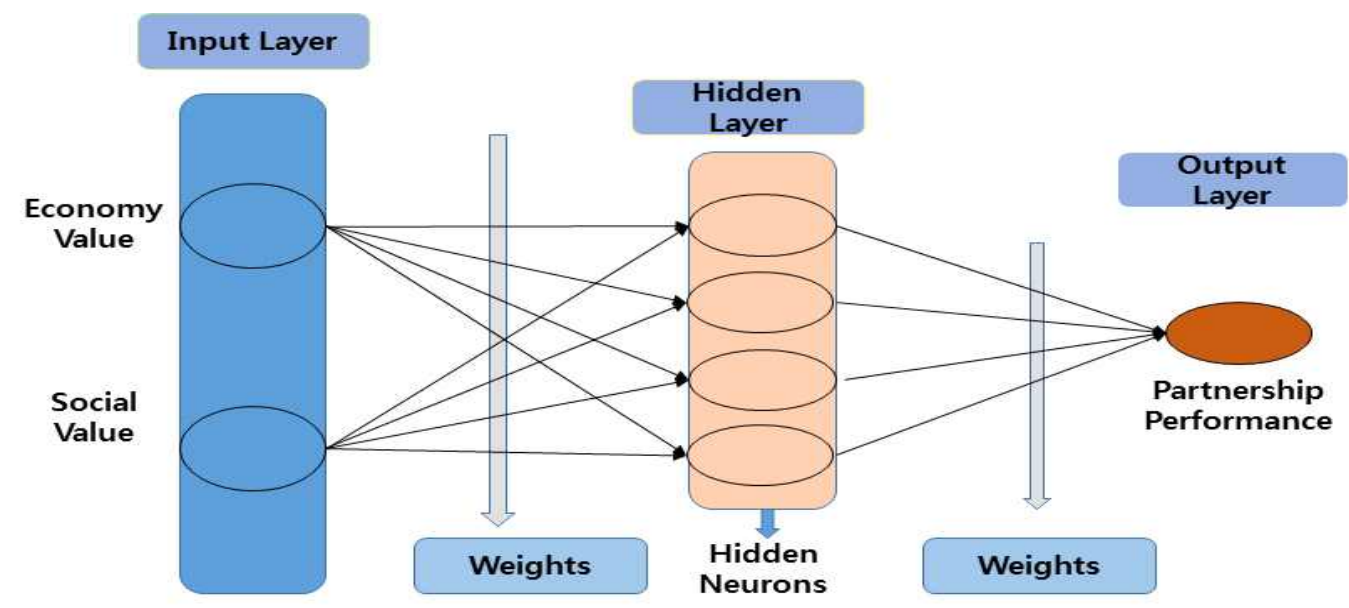

[Fig. 2] Research Model

\subsection{Method}

The data were collected through a survey of approximately 50 small and medium-sized firm employees and practitioners in the aviation manufacturers.

Based on the validity and reliability of all questionnaires applied to the variables in this study, ANN is a new analysis method that nonlinearly shows the relationship between variables, unlike a structural equation model that represents simple linear results[6]. Therefore, the hypothesis was verified through the ANN analysis method.

\subsection{Demographic Characteristics}

According to demographic characteristics, male participants accounted for $95.1 \%$ of the sample, while female respondents accounted for $4.9 \%$. This disproportion is due to the specificity of work in the aviation manufacturing industry. Regarding the number of working years, most participants $(62.6 \%)$ had over 15 years of experience, followed by $14.2 \%$ with $1-5$ years, $13.0 \%$ with $5-10$ years, $6.1 \%$ for less than 1 year, and $6.1 \%$ for less than $10-15$ years, respectively. As 

to the education degree, $73.6 \%$ of the participants were college graduates, and $10.9 \%$ had a degree higher that graduate school. Overall, the participants had a high education level (see Table 1).

[Table 1] Demographic Characteristics

\begin{tabular}{|c|c|c|c|}
\hline & Item & Frequency & Ratio (\%) \\
\hline \multirow{2}{*}{ Gender } & Male & 234 & 95.1 \\
\hline & Female & 12 & 4.9 \\
\hline \multirow{4}{*}{ Age } & $20^{\prime} \mathrm{s}$ & 33 & 13.4 \\
\hline & 30 's & 41 & 16.7 \\
\hline & $40^{\prime} \mathrm{s}$ & 64 & 26.0 \\
\hline & Over 50 & 108 & 43.9 \\
\hline \multirow{4}{*}{ Education } & High school & 25 & 10.2 \\
\hline & College & 13 & 5.3 \\
\hline & University & 181 & 73.6 \\
\hline & Graduated School & 27 & 10.9 \\
\hline \multirow{5}{*}{ Work experience } & Less than $1 y r$ & 15 & 6.1 \\
\hline & 1-less than5yr & 35 & 14.2 \\
\hline & 5-less than10yr & 32 & 13.0 \\
\hline & 10-less than15yr & 10 & 4.1 \\
\hline & Over 15yr & 154 & 62.6 \\
\hline \multirow{4}{*}{ Type of work } & Office work & 205 & 83.3 \\
\hline & Production line & 12 & 4.9 \\
\hline & Manager & 26 & 10.6 \\
\hline & Etc. & 3 & 1.2 \\
\hline \multirow{5}{*}{ Position } & General employee & 61 & 24.8 \\
\hline & Deputy of manager & 5 & 2.0 \\
\hline & Manger & 50 & 20.4 \\
\hline & Director & 28 & 11.4 \\
\hline & Etc. & 102 & 41.4 \\
\hline
\end{tabular}

\section{Results}

\subsection{Economic Value and Performance of Partner Companies}

As shown in [Fig. 3], economic value, a sub-factor of the creation of shared value, was found to have a positive effect on the performance of partner companies. Specifically, as the value of 
the economic value increased from 1.8 to 5.4 , the performance of partner companies also rose from 1.83 or higher to 5.0. The graph between the two variables also shows a curved graph, rather than a simple linear shape.

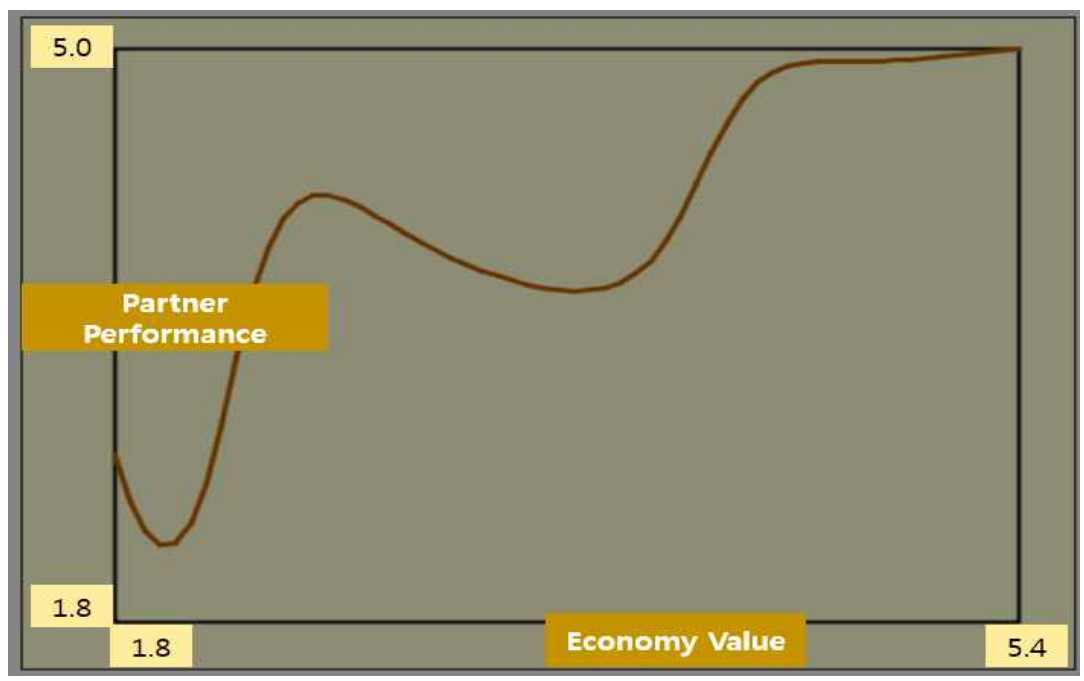

[Fig. 3] The Relationship between Economy CSV and Partner Company Performance

\subsection{Social Values and Performance of Partner Companies}

As shown in [Fig. 4], social value, a sub-factor of the creation of shared values, was found to have a positive effect on the performance of partner companies. Specifically, as the value of the economic value increased from 1.60 to 5.20 , the performance of partner companies initially had a negative effect from 1.83, and the social value increased from about 3.0 or higher to about 5.0. The results showed the actual relationship between the two variables with a curved graph, rather than a simple linear relationship shown in the regression model.

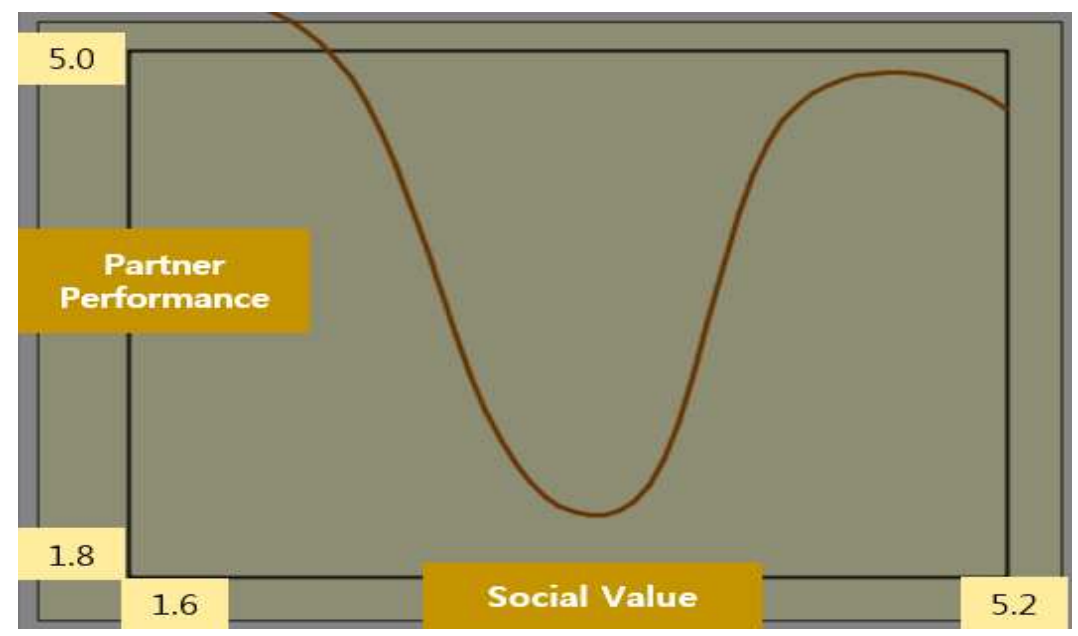

[Fig. 4] The Relationship between Social CSV and Partner Company Performance 


\section{Conclusions}

The theoretical and practical implications of this study are as follows.

First, we found that economic value activity, a sub-factor of the creation of shared values of large companies, has a positive effect on the performance of partner companies. These results are consistent with the results reported in previous studies. In other words, economic CSV creation activities, such as observing payment date, technical investment, and so forth, which are economic support activities of small- and medium-sized enterprises' subsidiaries, have a significant positive effect on the business performance of subcontractors.

Second, social value activity, which is a sub-factor of the creation of shared values of large companies, was found to have a positive effect on the performance of partner companies. Although the results of this study were similar to those reported in previous studies, they showed nonlinear studies different from those of the rare model or structural equation model, which shows the relationship between the variables in a linear graph.

Third, social value activities such as solving social-environmental problems of large companies and creating local employment were generally perceived to result in additional costs for companies; therefore, many companies hesitated about the introduction of corporate activities. However, as confirmed by the results of this study, this social value creation activity increases the management performance of partner companies and eventually leads to a virtuous cycle that leads to the improvement of quality and competitiveness of large companies in the long term

Therefore, instead of a corporate strategy focused on short-term economic performance, corporate decision makers should actively implement a CSV strategy that can create win-win values.

\section{Reference}

[1] De los Reyes Jr, G., \& Scholz, M., The limits of the business case for sustainability: Don't count on 'Creating Shared Value' to extinguish corporate destruction, Journal of Cleaner Production, (2019), Vol.221, pp.785-794.

[2] Cho Sang-Mi, Lee Jae-Hee, A Study on CSV Strategies with the Diamond Model - With a focus on Samsung Electronics, Korea Business Review, (2015), Vol.19, No.3, pp.35-58.

[3] Kwon Yung-Chul, A Study on Organizational Dynamic Capabilities to Create Shared Value and Its Theoretical Background, The Korean Association of Logos Management, (2016), Vol.14, No.4, pp.57-74.

[4] Michael E. Porter, Mark R. Kramer, Creating shared value, Harvard Business Review, https://www.communitylivingbc.ca/wp-content/uploads/2018/05/Creating-Shared-Value.pdf, March 1 (2011).

[5] Sekertekin, A., Arslan, N., Bilgili, M., Modeling diurnal Land Surface Temperature on a local scale of an arid environment using artificial Neural Network (ANN) and time series of Landsat-8 derived spectral indexes, Journal of Atmospheric and Solar-Terrestrial Physics, (2020), Vol.206, DOI: https://doi.org/10.1016/j.jastp.2020.105328

[6] Hasan, N. A., Methodological Approach for Predicting COVID-19 Epidemic Using EEMD-ANN Hybrid Model, Internet of Things, (2020), Vol.11, DOI: https://doi.org/10.1016/j.iot.2020.100228 
[7] Praveen, U., Farnaz, G., \& Hatim, G., Inventory management and cost reduction of supply chain processes using AI based time-series forecasting and ANN modeling, Procedia Manufacturing, (2019), Vol.38, pp.256-263, DOI: https://doi.org/10.1016/j.promfg.2020.01.034

[8] Steven Walczak, Artificial Neural Networks, Advanced Methodologies and Technologies in Artificial Intelligence, Computer Simulation, and Human-Computer Interaction, USA: IGI Global, (2019).

[9] Azarnoush Ansari, Arash Riasi, Modelling and evaluating customer loyalty using neural networks: Evidence from startup insurance companies, Future Business Journal, (2016), Vol.2, No.1, pp.15-30. 